\title{
A Local Market Mechanism for Physical Storage Rights
}

Thomas, Dimitrios; Kazempour, Jalal; Papakonstantinou, Athanasios; Pinson, Pierre; Deblecker, Olivier; loakimidis, Christos S.

Published in:

IEEE Transactions on Power Systems

Link to article, DOI:

10.1109/TPWRS.2020.2967998

Publication date:

2020

Document Version

Peer reviewed version

Link back to DTU Orbit

Citation (APA):

Thomas, D., Kazempour, J., Papakonstantinou, A., Pinson, P., Deblecker, O., \& loakimidis, C. S. (2020). A Local Market Mechanism for Physical Storage Rights. IEEE Transactions on Power Systems, 35(4), [8963661]. https://doi.org/10.1109/TPWRS.2020.2967998

\section{General rights}

Copyright and moral rights for the publications made accessible in the public portal are retained by the authors and/or other copyright owners and it is a condition of accessing publications that users recognise and abide by the legal requirements associated with these rights.

- Users may download and print one copy of any publication from the public portal for the purpose of private study or research.

- You may not further distribute the material or use it for any profit-making activity or commercial gain

- You may freely distribute the URL identifying the publication in the public portal

If you believe that this document breaches copyright please contact us providing details, and we will remove access to the work immediately and investigate your claim 


\title{
A Local Market Mechanism for Physical Storage Rights
}

\author{
Dimitrios Thomas, Student Member, IEEE, Jalal Kazempour, Senior Member, IEEE, \\ Athanasios Papakonstantinou, Pierre Pinson, Fellow, IEEE, \\ Olivier Deblecker, Member, IEEE, Christos S. Ioakimidis, Member, IEEE
}

\begin{abstract}
This paper proposes a two-stage auction-based local market mechanism to allocate physical storage rights (PSRs). As a market product, PSRs are provided by a storage owner and enable the local market participants (including renewable producers, consumers and prosumers) to access the storage. That is, they can book storage in the form of PSRs and dispatch it at a given time aiming to maximize their utility function. The business options we examine to evaluate the position of storage in the market range from storage owner entirely participating in day-ahead (DA) and real-time (RT) markets as an intertemporal arbitrager, to exclusively acting as a PSR provider in DA only - this way, the storage owner is fully paid upfront in DA. Considering the context above, we propose an equilibrium model where each player optimizes its operational objective. We prove that the equilibrium model can be substituted with an equivalent optimization formulation which clears the proposed market ensuring the same desirable market properties, such as efficiency and revenue adequacy. Results suggest that the certain revenues earned by the storage owner in DA when acting as a PSR provider is equal to its expected profit as a regular market participant, mitigating however its payoff uncertainty and resulting in the same economic return.
\end{abstract}

Index Terms- Energy storage, local market design, physical storage rights, equilibrium, optimization

\section{INTRODUCTION}

E NERGY storage (ES) can improve power system economics and reliability by providing various market remunerated and regulated services. The market remunerated services include among others ancillary services (balancing and contingency), energy services (ES can participate in energy markets by arbitraging electricity prices), and capacity services (ES can provide capacity similar to traditional generators, reducing the need for new generation investment) [1], [2]. Regulated services refer to grid infrastructure investment deferral. ES can support transmission and distribution systems by mitigating congestion and improving power quality [1].

Depending on storage ownership, it can be either a merchant or a system asset. As a system asset, it provides services

D. Thomas and O. Deblecker are with the Electrical Power Engineering Unit, University of Mons, Mons, 7000 Belgium (e-mail: dimitrios.thomas@umons.ac.be; olivier.deblecker@umons.ac.be).

J. Kazempour and P. Pinson are with the Department of Electrical Engineering, Technical University of Denmark, Kgs. Lyngby, 2800 Denmark (e-mail: seykaz@elektro.dtu.dk; ppin@elektro.dtu.dk).

A. Papakonstantinou is with the Department of Technology, Management and Economics, Technical University of Denmark, Kgs. Lyngby, 2800 Denmark (e-mail: athpapa@dtu.dk)

C. S. Ioakimidis is with the ERA Chair 'Net-Zero Energy Efficiency on City Districts', Research Institute for Energy, University of Mons, Mons, 7000 Belgium (e-mail: christos.ioakeimidis@umons.ac.be)

This work was supported by EC under the FP7 RESIZED 621408 (Research Excellence for Solutions and Implementation of Net-Zero Energy City Districts) project. to support the whole system, e.g., peak shaving, voltage profile improvement, ancillary service provision, increasing the reliability of the system to meet critical loads [3], etc. To this end, the work in [4] proposes an ES sizing approach considering wind uncertainty to minimize the total power system cost and improve voltage profiles. In [5], installation of ES is used as a possible voltage quality remedy in weak distribution networks with increased wind penetration. The proposed ES control aims at the system's short-term voltage quality improvement.

On the contrary, a merchant storage belongs to a market participant who seeks to maximize its profit (or minimize its cost) by directly arbitraging energy over time periods. In [6], a merchant ES acts as a price-maker to maximize its expected profit by determining the most beneficial trading actions in day-ahead and balancing pool-based markets under real-time net load uncertainty. Dictorato et al. [7] propose the planning and operation of a combined wind-storage system which uses ES to compensate for wind variability and maximize wind farm's expected profit. The operation of an ES providing ancillary services to the system, such as frequency regulation service, spinning and non-spinning reserves, etc., is examined in [8]. The proposed robust and stochastic optimization approaches take into account multiple uncertainties aiming at the estimation of the ES potential profit. Existing literature also addresses the action of merchant-oriented storage facilities in a strategic way. To this end, [9] proposes a model where the ES facility seeks to maximize its profit through strategic planning, i.e., storage sizing, strategic offering and bidding decisions. Similarly, a strategic bidding behaviour implemented in [10] seeks to maximize the expected profit of a virtual plant, which among others includes an ES facility, using robust optimization to deal with renewable production and market price uncertainties.

The commercial availability of small battery capacities up to $100 \mathrm{kWh}$ (e.g., Tesla Powerpack) intended for smallscale usage unfolds a new potential on sharing ES capacity in local markets with prosumer communities. Storage could be used for load shifting, backup power, demand response, renewable power integration, etc. The focus of this paper is on storage assets offered for merchant-related purposes to the market participants, located in local energy communities [11]. However, apart from the direct way of profiting for the storage owners (e.g., by arbitraging energy prices in energy markets), we also investigate the possibility of storage owners profiting indirectly from the provision of an additional market product, whose value depends on price variations. The motivation of this work is to explore business models for small-scale 
merchant storage systems through interaction with other local market participants (e.g., renewables producers, prosumers and consumers).

We specifically investigate a market product, the so-called physical storage right (PSR), which enables local market participants to access the storage, but in a systematic marketbased manner. Using the PSRs, market participants can book storage and dispatch it at a given time aiming at maximizing their own profit (or minimizing their cost). The potential PSR holders do not necessarily have to bid for the entire ES asset; they can place bids for partially booking ES to the extent their needs are met. Note that these players need to bid separately to earn access to charging, discharging and reservoir devices of an ES. Therefore, PSRs include charging, discharging and capacity rights. At the same time, storage owners obtain an additional option for commercializing their ES assets apart from participating in the market as regular arbitrageurs.

Financial storage rights (FSRs), i.e., another type of rights that are mainly encountered at wholesale level, allow the market operator to access the storage asset and dispatch it, aiming at minimizing the system cost. The merchandising surplus in this case (e.g., the storage congestion rent) is then distributed among the financial right holders [12], [13]. Both FSRs and PSRs are market products that can lead to business models. However, FSRs treat ES as a system asset managed by the market operator. On the other hand, market participants can obtain entire control of their booked ES through the acquisition of PSRs.

A preliminary work introducing the concept of allocating storage rights is carried out in [14] where the proposed business model aggregates multiple revenue streams of ES by coordinating a series of auctions. In these auctions, the right to utilize the ES is determined upon different time horizons. The first systematic study to implement PSRs is performed in [15] where a framework for shared ES resources through a periodically organized auction is proposed. In addition, [16] introduces the use of storage-capacity rights to surmount the regulatory and cost-recovery issues that ES faces. These issues are mainly associated with the multiple services storage can provide and with some regulatory authorities suggesting that storage developers must choose between classifying their assets as providing competitively priced or unpriced services [16]. The proposed solution disengages storage cost recovery from the regulatory treatment of its end-use, demonstrating how to price storage capacity rights efficiently.

The studies mentioned above refer to deterministic setups without taking uncertainty into account, which is endemic in local energy communities. Specifically, by uncertainty we refer to renewables and electricity price unpredictability. However, uncertainty especially in such small-scale local market is a key point, and it is of high interest from an ES perspective to explore its potential business models in a two-stage setup which links day-ahead (DA) scheduling with real-time (RT) operation. The available business options for storage range from entirely participating in DA and RT markets as an intertemporal arbitrager, to exclusively acting as a PSR provider in DA only - this way, the storage owner is fully paid upfront in $\mathrm{DA}$, and its payoff in RT is zero. In this paper, we investigate the impact of these market participation choices on storage owner's profit (in expectation and its standard deviation), while at the same time we also explore the social impacts deriving from each choice, e.g., effect on local system cost, and the payoff of other market participants.

Considering the context above, the main contributions of this paper are as follows. We propose a two-stage auctionbased market-clearing setup to design an efficient platform for trading PSRs in a local energy market under uncertainty. The resulting model is a Nash equilibrium problem (NEP), i.e., a set of interconnected optimization problems in which each market participant maximizes its expected profit. We then prove that it is possible to exactly reformulate this equilibrium model with a more computationally tractable optimization problem ensuring the same desirable economic properties in the market. That is (i) market efficiency, which means that the system is dispatched at the minimum social cost and no one is motivated to unilaterally deviate from the marketclearing outcomes, and (ii) revenue adequacy, which implies that the market operator never incurs a budget deficit. All the necessary proofs are provided in the online Appendix [17] and in Appendix B.

Numerical results suggest that for a storage owner, its certain DA profit as a PSR provider is equal to its expected profit as an arbitrageur in DA and RT markets, reducing its profit uncertainty and offering a possibility to hedge against RT price volatility. While the total system cost decreases with the introduction of storage in either form, when ES operates as a PSR provider, the majority of market participants gets involved more actively in RT market. We numerically verify the aforementioned remarks by performing an ex-post outof-sample simulation. Moreover, we take the grid constraint (i.e., the one connecting the local structure to the distribution system) into account evaluating how a possible line congestion could cause additional system cost.

The remainder of this paper is structured as follows. Section III provides preliminaries on the general structure of the market including the local setup and the market participants, and then lists our assumptions. Section III presents the mathematical formulation for the proposed equilibrium and optimization market models and explains why they are equivalent. Section IV]provides a comprehensive case study based on two different business options for storage utilization. Section $\mathrm{V}$ concludes the paper. Appendix A presents the nomenclature. The revenue adequacy of the proposed local market is proven in Appendix $\mathrm{B}$, while Appendix $\mathrm{C}$ provides technical information required throughout the paper.

\section{General Structure And Assumptions}

\section{A. Market Participants and Preliminaries}

The following market participants are considered in constructing the equilibrium and the optimization models: individuals arbitraging DA and RT market prices; wind producers focusing on portfolio management; consumers; prosumers (i.e., a customer that can consume but also produce energy); the grid 
operator; and the storage owner ${ }^{1}$. The arbitrageur (either as a storage owner or as a PSR holder) seeks to benefit from hourly price differences in the DA and RT market. The wind producer can either inject its produced energy back to the grid and/or use the ES resources to increase the market value of its renewable generation. The consumer, whose daily load must be served, could use the ES for peak shaving and/or load shifting. It could, for example, defer some of its peak period consumption by charging the ES during periods when electricity is cheap, and by discharging it during peak periods when electricity price is high. The prosumer functions in a similar way with the consumer being able though to produce a small amount of energy which usually comes from photovoltaic (PV). The grid owner, for instance the distribution system operator, has no access to the shared storage resources but it can benefit when the network is congested by performing spatial arbitrage between the local market and the distribution network.

To operate the local market, we propose a non-profit entity such as a local community manager [18] which would be responsible for clearing the market. That is, it would decide for the optimal dispatch of the production and consumption resources, the energy storage charge/discharge scheduling profiles, as well as the DA allocation of the PSRs. In addition, the interaction with the grid owner (either this is the distribution system operator or some other entity) assumes the communication to the community manager of information such as the capacity of lines. On the contrary, the interaction of the community manager with the wholesale market operator is only price-wise, as we assume that the distribution-level price reflects the wholesale market price.

Note that we do not propose a new market for the PSRs, on top of the energy market. On the contrary, we suggest the integration of a new market product (i.e., the PSRs) to the existing market operation. Thus, the duties of the community manager would be in line with the duties of the existing market operators $^{2}$

We propose a local market design not due to a fundamental limitation emerging from wholesale level but because we envision that such local markets could soon emerge [11]. The consideration of storage in local communities offers access to ES resources to smaller individuals (a household, a small business, etc.) broadening their energy options.

\section{B. The Local Market Setup}

The market participants described in section II-A are either profit maximizers (arbitrageurs, wind producers and storage owner) or cost minimizers (consumers and prosumers). They formulate a NEP, where each player aims to optimize the value of its objective function taking the decisions of all rivals into

\footnotetext{
${ }^{1}$ In this paper we assume that, depending on the business case, storage is either owned or offered exclusively by a single entity. However, it is possible to modify the proposed market mechanism so as storage is owned by multiple market participants. This way, in addition to directly using storage for their own interest, the market participants who own storage would further seek to indirectly maximize their profit (or minimize their cost) by providing their unused storage capacity in the form of PSRs as an additional market product.

${ }^{2}$ The proposed local market is revenue adequate, as proven in Appendix B. Therefore, the market operator (the local community manager in our case) never incurs a financial deficit.
}

account. The hourly market clearing process results, for both models, in a single uniform PSR price per storage offered. One should also note that this is characterized as a non-cooperative game, as they are driven by the competition between individual players. We describe below the equilibrium and optimization models of the local market model setun ${ }^{3}$

Equilibrium model: In this two-stage stochastic NEP, the decision process of each player is represented through its particular optimization problem which is subject to its individual constraints. That is, each participant optimizes its payoff, which is a function of energy and PSR prices. These prices are parameters in their problems, while they are variables for the whole equilibrium problem. The equilibrium model embodies all optimization problems, one per participant, as well as sharing constraints which impose balance equalities for energy and rights.

Optimization model: This is a two-stage stochastic model, where the market operator solves a two-stage stochastic problem minimizing the total expected system cost, while scheduling the PSRs provision, the storage power profile, and the generation/demand in both DA and RT. The constraints of the market participants in this model are identical to those of Equilibrium model.

\section{Assumptions}

We now review some general assumptions about the market participants' behaviour, information and costs. All participants act competitively, and they are assumed to be price takers with a common underlying probability distribution of the uncertainty. We also make the simplifying assumption that in the RT market, the real-time conditions for the entire day, such as hourly wind, prosumer's PV production, and RT prices, are represented by a set of possible scenarios $(\omega \in \Omega)$ with their corresponding probability $\pi_{\omega}$. While the set of scenarios and their probabilities are known in DA, which one actually occurs in RT is not. This is a common approach in literature to provide a sufficient representation of uncertainty in two-stage optimization programming [19]. In terms of modeling, this implies that all sources of uncertainty have been aggregated into one. Moreover, although technically simultaneous charge and discharge actions are allowed within our models, due to the different (dis)charge efficiencies considered and the optimization functions, we prompt (dis)charge variables not taking non-zero values at the same time step. The use of auxiliary binary variables contributes to a more accurate modeling of the ES dis(charge) process but does not maintain convexity which is necessary in our study to derive optimality conditions. This approach is commonly followed in literature [15], [20]. We also exclude the grid within the local market, and consider a single connection between the whole local market and the distribution system, managed by a grid operator. Lastly, although in practice retail electricity rates tend

\footnotetext{
${ }^{3}$ We prove later that the proposed equilibrium and the optimization models are equivalent, and thus they hold the same market properties. However, they are not equivalent from an information sharing point of view. Indeed, optimization problems with centrally defined objectives require from agents to reveal some of their information to the market operator (e.g., production/demand curves, DA forecasts/RT scenarios, nominal values of storage, capacity of distribution lines, etc.).
} 
to be highly regulated, different across types of distribution customers, and varied depending on the utility provider, for the sake of simplicity we assume that all market participants face the same (marginal) electricity rates.

\section{MATHEMATICAL FORMULATION}

The mathematical formulation of the two local market models is presented in this section. Although all notation are defined throughout the paper, a nomenclature is provided in Appendix A for further clarity. Note also that dual variables are listed alongside each constraint.

\section{A. Equilibrium Model}

In Equilibrium Model, comprising (1a)-(1g), six sets of optimization problems (one per market participant) and the set of sharing constraints are provided. First, we present the optimization problem of the purely financial arbitrageur $i \in \mathcal{A}$, who owns no physical asset:

$$
\begin{aligned}
& \left\{\underset { \Xi _ { \mathcal { A } } } { \operatorname { M a x i m i z e } } \sum _ { t , s } \left[\lambda_{t}^{\mathrm{loc}, \mathrm{DA}}\left(p_{i, s, t}^{d, \mathrm{DA}}-p_{i, s, t}^{c, \mathrm{DA}}\right)-p_{i, s, t}^{c, \max , \mathrm{DA}} \mu_{s, t}^{c, \mathrm{DA}}\right.\right. \\
& \left.-p_{i, s, t}^{d, \max , \mathrm{DA}} \mu_{s, t}^{d, \mathrm{DA}}-e_{i, s, t}^{\max , \mathrm{DA}} \mu_{s, t}^{e, \mathrm{DA}}\right]+\sum_{s} \tilde{\lambda} e_{i, s, T}^{\mathrm{DA}} \\
& +\sum_{t, s, \omega} \pi_{\omega}\left[\frac{\lambda_{t, \omega}^{\mathrm{loc}, \mathrm{RT}}}{\pi_{\omega}}\left(p_{i, s, t, \omega}^{d, \mathrm{RT}}-p_{i, s, t, \omega}^{c, \mathrm{RT}}\right)\right]+\sum_{s, \omega} \pi_{\omega} \tilde{\lambda} e_{i, s, T, \omega}^{\mathrm{RT}}
\end{aligned}
$$

subject to:

$$
\begin{aligned}
& 0 \leq p_{i, s, t}^{c, \mathrm{DA}} \leq p_{i, s, t}^{c, \max , \mathrm{DA}}: \underline{\theta}_{i, s, t}^{c, \mathrm{DA}}, \bar{\theta}_{i, s, t}^{c, \mathrm{DA}} \forall t, s \\
& 0 \leq p_{i, s, t}^{d, \mathrm{DA}} \leq p_{i, s, t}^{d, \mathrm{max}, \mathrm{DA}}: \underline{\theta}_{i, s, t}^{d, \mathrm{DA}}, \bar{\theta}_{i, s, t}^{d, \mathrm{DA}} \forall t, s \\
& 0 \leq e_{i, s, t}^{\mathrm{DA}} \leq e_{i, s, t}^{\max , \mathrm{DA}}: \underline{\theta}_{i, s, t}^{e, \mathrm{DA}}, \bar{\theta}_{i, s, t}^{e, \mathrm{DA}} \forall t, s \\
& e_{i, s, t}^{\mathrm{DA}}=E_{i, s}^{\mathrm{ini}}+p_{i, s, t}^{c, \mathrm{DA}} \eta_{s}^{c}-\frac{p_{i, s, t}^{d, \mathrm{DA}}}{\eta_{s}^{d}}: \gamma_{i, s, t}^{e, \mathrm{DA}} \forall s, t=1 \\
& e_{i, s, t}^{\mathrm{DA}}=e_{i, s, t-1}^{\mathrm{DA}}+p_{i, s, t}^{c, \mathrm{DA}} \eta_{s}^{c}-\frac{p_{i, s, t}^{d, \mathrm{DA}}}{\eta_{s}^{d}}: \gamma_{i, s, t}^{e, \mathrm{DA}} \forall s, t>1 \\
& 0 \leq\left(p_{i, s, t}^{c, \mathrm{DA}}+p_{i, s, t, \omega}^{c, \mathrm{RT}}\right) \leq p_{i, s, t}^{c, \max , \mathrm{DA}}: \underline{\theta}_{i, s, t, \omega}^{c, \mathrm{RT}}, \bar{\theta}_{i, s, t, \omega}^{c, \mathrm{RT}} \\
& 0 \leq\left(p_{i, s, t}^{d, \mathrm{DA}}+p_{i, s, t, \omega}^{d, \mathrm{RT}}\right) \leq p_{i, s, t}^{d, \max , \mathrm{DA}}: \underline{\theta}_{i, s, t, \omega}^{d, \mathrm{RT}}, \bar{\theta}_{i, s, t, \omega}^{d, \mathrm{RT}} \\
& 0 \leq\left(e_{i, s, t}^{\mathrm{DA}}+e_{i, s, t, \omega}^{\mathrm{RT}}\right) \leq e_{i, s, t}^{\max , \mathrm{DA}}: \underline{\theta}_{i, s, t, \omega}^{e, \mathrm{RT}}, \bar{\theta}_{i, s, t, \omega}^{e, \mathrm{RT}} \forall t, s, \omega \\
& e_{i, s, t, \omega}^{\mathrm{RT}}=p_{i, s, t, \omega}^{c, \mathrm{RT}} \eta_{s}^{c}-\frac{p_{i, s, t, \omega}^{d, \mathrm{RT}}}{\eta_{s}^{d}}: \gamma_{i, s, t, \omega}^{e, \mathrm{RT}} \forall s, \omega, t=1 \\
& e_{i, s, t, \omega}^{\mathrm{RT}}=e_{i, s, t-1, \omega}^{\mathrm{RT}}+p_{i, s, t, \omega}^{c, \mathrm{RT}} \eta_{s}^{c}-\frac{p_{i, s, t, \omega}^{d, \mathrm{RT}}}{\eta_{s}^{d}}: \gamma_{i, s, t, \omega}^{e, \mathrm{RT}} \\
& \forall s, \omega, t>1\} \forall i \in \mathcal{A}
\end{aligned}
$$

where $\Xi_{\mathcal{A}}=\left\{p_{i, s, t}^{c, \mathrm{DA}}, p_{i, s, t}^{d, \mathrm{DA}}, e_{i, s, t}^{\mathrm{DA}}, p_{i, s, t}^{c, \max , \mathrm{DA}}, p_{i, s, t}^{d, \max , \mathrm{DA}}\right.$, $\left.e_{i, s, t}^{\max , \mathrm{DA}}, p_{i, s, t, \omega}^{c, \mathrm{RT}}, p_{i, s, t, \omega}^{d, \mathrm{RT}}, e_{i, s, t, \omega}^{\mathrm{RT}}\right\}$ is the set of primal variables. The arbitrageur aims to use storage resources to take advantage of price differences over time. The access to ES is achieved through the acquisition of PSRs.

Objective function 1aa maximizes the profit of the arbitrageur. More specifically, it buys charge right $p_{i, s, t}^{c, \max , \mathrm{DA}}$, discharge right $p_{i, s, t}^{d, \max , \mathrm{DA}}$ and capacity right $e_{i, s, t}^{\max , \mathrm{DA}}$ at time $t$ from storage $s$ at prices $\mu_{s, t}^{c, \mathrm{DA}}, \mu_{s, t}^{d, \mathrm{DA}}$ and $\mu_{s, t}^{e, \mathrm{DA}}$ in DA market, respectively. Note that these prices are market outcomes (variables for the equilibrium), while parameters within (1a). One should also notice that the obtained PSRs operate as the upper bounds for the maximum levels of charging, discharging and energy capacity of storage $s$ booked by the arbitrageur.

Subsequently, according to the total PSRs obtained in DA, the arbitrageur can exploit it in both DA and RT. In DA, it charges power $p_{i, s, t}^{c, \mathrm{DA}}$ or discharges power $p_{i, s, t}^{d, \mathrm{DA}}$ at local price $\lambda_{t}^{\text {loc,DA }}$. In RT, it is able to modify the final ES (dis)charge quantities by adjusting the charge $p_{i, s, t, \omega}^{c, \mathrm{RT}}$ or discharge $p_{i, s, t, \omega}^{d, \mathrm{RT}}$ free variables at price $\frac{\lambda_{t, \omega}^{\mathrm{loc}, \mathrm{RT}}}{\pi_{\omega}}$. Note that $\frac{\lambda_{t, \omega}^{\mathrm{loc}, \mathrm{RT}}}{\pi_{\omega}}$ refers to the RT probability-adjusted local market price at time $t$ under scenario $\omega$. This price is obtained by dividing each dual variable $\lambda_{t, \omega}^{\text {loc,RT }}$ by its associated probability $\pi_{\omega}$. This way, the DA local market prices and the RT probability-adjusted local prices are of the same order of magnitude [19], [21]. The local prices $\lambda_{t}^{\mathrm{loc}, \mathrm{DA}}$ and $\frac{\lambda_{t, \omega}^{\mathrm{loc}, \mathrm{RT}}}{\pi_{\omega}}$, like the PSR prices, are variables for the equilibrium, while paremeters within (1a). Unlike most studies where residual energy at the end of the scheduling horizon is either kept equal to the initial state-ofcharge (SOC) or anchored within an acceptable deviation from initial level, the concept of energy value is used here to reflect the value of the residual energy in ES. The residual energy has the potential to yield profits by generating (discharging) in the following horizons. Therefore, a price $\tilde{\lambda}$ is assigned to the final energy level (state-of-energy) of the ES at the end of the time horizon considered, i.e., $t=T$ to reflect its value [22]. The resulting energy value is considered in the objective function. Constraints $1 \mathrm{ab}-1 \mathrm{ad}$ impose that the actual storage (dis)charge power and the SOC in DA are nonnegative and bounded by the corresponding PSR variables. Storage day-ahead SOC $e_{i, s, t}^{\mathrm{DA}}$ is calculated by (1ae-1af). Parameter $E_{i, s}^{\text {ini }}$ expresses the initial SOC and $\eta_{s}^{c}, \eta_{s}^{d}$ are the (dis)charge efficiencies for storage $s$. Constraints 1ag -1 1ai state that the adjusted storage (dis)charge power and SOC in RT must be non-negative and respect the corresponding dayahead PSR variables. Finally, constraints 1aj-1ak calculate the adjusted real-time $\mathrm{SOC} e_{i, s, t, \omega}^{\mathrm{RT}}$.

Likewise, the profit-maximization problem for each wind producer $^{4} i \in \mathcal{P}$ is given by (1b) below. This market participant does not own any ES, but it is willing through the acquisition of PSRs to gain access to ES and increase the market value of its renewable generation:

$$
\left\{\underset { \Xi _ { \mathcal { P } } } { \operatorname { M a x i m i z e } } \sum _ { t } \left[\lambda_{t}^{\mathrm{loc}, \mathrm{DA}} p_{i, t}^{g, \mathrm{DA}}+\sum_{s}\left(\lambda_{t}^{\mathrm{loc}, \mathrm{DA}} p_{i, s, t}^{d, \mathrm{DA}}\right.\right.\right.
$$

${ }^{4}$ Optimization $1 \mathrm{~b}$ can be extended to cover other types of intermittent renewable energy sources, as long as a sufficient number of scenarios is selected in order to adequately describe the underlying uncertainty, as opposed to non-intermittent, which due to their deterministic nature can be trivially modeled. 


$$
\begin{aligned}
& \left.\left.-p_{i, s, t}^{c, \max , \mathrm{DA}} \mu_{s, t}^{c, \mathrm{DA}}-p_{i, s, t}^{d, \max , \mathrm{DA}} \mu_{s, t}^{d, \mathrm{DA}}-e_{i, s, t}^{\max , \mathrm{DA}} \mu_{s, t}^{e, \mathrm{DA}}\right)\right] \\
& +\sum_{s} \tilde{\lambda} e_{i, s, T}^{\mathrm{DA}}+\sum_{t, \omega} \pi_{\omega}\left[\frac{\lambda_{t, \omega}^{\mathrm{loc}, \mathrm{RT}}}{\pi_{\omega}}\left(p_{i, t, \omega}^{g, \mathrm{RT}}+\sum_{s} p_{i, s, t, \omega}^{d, \mathrm{RT}}\right)\right] \\
& +\sum_{s, \omega} \pi_{\omega} \tilde{\lambda} e_{i, s, T, \omega}^{\mathrm{RT}}
\end{aligned}
$$

subject to:

$$
\begin{aligned}
& 1 \mathrm{ab}-1 \mathrm{ak} \\
& 0 \leq p_{i, t}^{g, \mathrm{DA}} \leq G_{i, t}^{\mathrm{DA}}-\sum_{s} p_{i, s, t}^{c, \mathrm{DA}}: \underline{\theta}_{i, t}^{g, \mathrm{DA}}, \bar{\theta}_{i, t}^{g, \mathrm{DA}} \forall t \\
& 0 \leq\left(p_{i, t}^{g, \mathrm{DA}}+p_{i, t, \omega}^{g, \mathrm{RT}}\right) \leq G_{i, t, \omega}^{\mathrm{RT}}-\sum_{s}\left(p_{i, s, t}^{c, \mathrm{DA}}+p_{i, s, t, \omega}^{c, \mathrm{RT}}\right) \\
& \left.: \underline{\theta}_{i, t, \omega}^{g, \mathrm{RT}}, \bar{\theta}_{i, t, \omega}^{g, \mathrm{RT}} \forall t, \omega\right\} \forall i \in \mathcal{P}
\end{aligned}
$$

where $\Xi_{\mathcal{P}}=\left\{p_{i, s, t}^{c, \mathrm{DA}}, p_{i, s, t}^{d, \mathrm{DA}}, e_{i, s, t}^{\mathrm{DA}}, p_{i, s, t}^{c, \max , \mathrm{DA}}, p_{i, s, t}^{d, \max , \mathrm{DA}}\right.$, $\left.e_{i, s, t}^{\max , \mathrm{DA}}, p_{i, s, t, \omega}^{c, \mathrm{RT}}, p_{i, s, t, \omega}^{d, \mathrm{RT}}, e_{i, s, t, \omega}^{\mathrm{RT}}, p_{i, t}^{g, \mathrm{DA}}, p_{i, t, \omega}^{g, \mathrm{RT}}\right\}$ is the set of primal variables. Constraint (1bc) implies that the produced wind power $\left(G_{i, t}^{\mathrm{DA}}\right)$ can either directly be sold to the grid $\left(p_{i, t}^{g, \mathrm{DA}}\right)$ or stored in the $\mathrm{ES}\left(p_{i, s, t}^{c, \mathrm{DA}}\right)$ whose rights are being bought in DA. Note that unlike the rest of market participants, the charging power potential of the wind producer at every time step is bounded not only from PSR constraints, but also from its maximum wind production. This way, the wind producer uses only its renewable, "cost-free" energy to charge the storage. Constraint (1bd) enforces that the total adjusted power to be sold to the grid $\left(p_{i, t}^{g, \mathrm{DA}}+p_{i, t, \omega}^{g, \mathrm{RT}}\right)$ in RT must be less than the underlying wind production scenario realization minus the total adjusted power $\left(p_{i, s, t}^{c, \mathrm{DA}}+p_{i, s, t, \omega}^{c, \mathrm{RT}}\right)$ to be charged in all ES. Note that (1bd) implicitly allows the excessive wind power to be spilled at no cost.

Furthermore, the cost-minimization problem for each consumer $i \in \mathcal{C}$ is given by (1c). Similarly, no ES belongs to this consumer, but it bids to buy PSRs from the storage owners:

$$
\begin{aligned}
& \left\{\underset { \Xi _ { \mathcal { C } } } { \operatorname { M i n i m i z e } } \sum _ { t } \left[-\lambda_{t}^{\mathrm{loc}, \mathrm{DA}} q_{i, t}^{\mathrm{DA}}\right.\right. \\
& +\sum_{s}\left(p_{i, s, t}^{c, \max , \mathrm{DA}} \mu_{s, t}^{c, \mathrm{DA}}+p_{i, s, t}^{d, \max , \mathrm{DA}} \mu_{s, t}^{d, \mathrm{DA}}\right. \\
& \left.\left.+e_{i, s, t}^{\max , \mathrm{DA}} \mu_{s, t}^{e, \mathrm{DA}}\right)\right]-\sum_{s} \tilde{\lambda} e_{i, s, T}^{\mathrm{DA}}-\sum_{s, \omega} \pi_{\omega} \tilde{\lambda} e_{i, s, T, \omega}^{\mathrm{RT}} \\
& +\sum_{t, \omega} \pi_{\omega}\left(-\frac{\lambda_{t, \omega}^{\mathrm{loc}, \mathrm{RT}}}{\pi_{\omega}} q_{i, t, \omega}^{\mathrm{RT}}+V_{i} p_{i, t, \omega}^{\mathrm{shed}}\right)
\end{aligned}
$$

subject to:

$$
\begin{aligned}
& 1 \mathrm{ab}-1 \mathrm{ak} \\
& 0 \leq p_{i, t, \omega}^{\mathrm{shed}} \leq D_{i, t}: \underline{\delta}_{i, t, \omega}, \bar{\delta}_{i, t, \omega} \forall t, \omega \\
& q_{i, t}^{\mathrm{DA}}=\sum_{s}\left(p_{i, s, t}^{d, \mathrm{DA}}-p_{i, s, t}^{c, \mathrm{DA}}\right)-D_{i, t}: \gamma_{i, t}^{q, \mathrm{DA}} \forall t \\
& q_{i, t}^{\mathrm{DA}}+q_{i, t, \omega}^{\mathrm{RT}}=\sum_{s}\left(p_{i, s, t}^{d, \mathrm{DA}}+p_{i, s, t, \omega}^{d, \mathrm{RT}}-p_{i, s, t}^{c, \mathrm{DA}}-p_{i, s, t, \omega}^{c, \mathrm{RT}}\right.
\end{aligned}
$$

$$
\left.+p_{i, t, \omega}^{\text {shed }}-D_{i, t}: \gamma_{i, t, \omega}^{q, \mathrm{RT}} \forall t, \omega\right\} \forall i \in \mathcal{C}
$$

where the set of primal variables is denoted by $\Xi_{\mathcal{C}}=$ $\left\{p_{i, s, t}^{c, \mathrm{DA}}, p_{i, s, t}^{d, \mathrm{DA}}, e_{i, s, t}^{\mathrm{DA}}, p_{i, s, t}^{c, \max , \mathrm{DA}}, p_{i, s, t}^{d, \max , \mathrm{DA}}, e_{i, s, t}^{\max , \mathrm{DA}}, p_{i, s, t, \omega}^{c, \mathrm{RT}}\right.$, $\left.p_{i, s, t, \omega}^{d, \mathrm{RT}}, e_{i, s, t, \omega}^{\mathrm{RT}}, q_{i, t}^{\mathrm{DA}}, q_{i, t, \omega}^{\mathrm{RT}}, p_{i, t, \omega}^{\mathrm{shed}}\right\}$. Constraint 1 1cc binds the total shedding power $\left(p_{i, t, \omega}^{\text {shed }}\right)$ to be non-negative and less than or equal to the total load $\left(D_{i, t}\right)$. Constraint $(1 \mathrm{~cd})$ defines the consumer's net energy generation $q_{i, t}^{\mathrm{DA}}$ (if positive) and consumption (if negative) in DA. Variable $q_{i, t, \omega}^{\mathrm{RT}}$ expresses the adjusted energy generation in RT. Parameter $V_{i}$ declares the value of lost load. Note that the total load $D_{i, t}$ is not scenario-dependent and that only the net energy variables $q_{i, t}^{\mathrm{DA}}, q_{i, t, \omega}^{\mathrm{RT}}$ are directly associated with the local electricity prices in the objective function of the consumer.

In addition, the cost-minimization problem of each prosumer $i \in \mathcal{R}$ who is willing to buy PSRs is given by $1 \mathrm{~d}$ below:

$\left\{\underset{\Xi_{\mathcal{R}}}{\operatorname{Minimize}}\right.$

subject to:

$1 \mathrm{ab}-1 \mathrm{ak} ; 1 \mathrm{cc}$

$0 \leq p_{i, t}^{\mathrm{PV}, \mathrm{DA}} \leq G_{i, t}^{\mathrm{DA}}: \underline{\rho}_{i, t}^{\mathrm{DA}}, \bar{\rho}_{i, t}^{\mathrm{DA}} \forall t$

$0 \leq p_{i, t}^{\mathrm{PV}, \mathrm{DA}}+p_{i, t, \omega}^{\mathrm{PV}, \mathrm{RT}} \leq G_{i, t, \omega}^{\mathrm{RT}}: \underline{\rho}_{i, t, \omega}^{\mathrm{RT}}, \bar{\rho}_{i, t, \omega}^{\mathrm{RT}} \forall t, \omega$

$q_{i, t}^{\mathrm{DA}}=\sum_{s}\left(p_{i, s, t}^{d, \mathrm{DA}}-p_{i, s, t}^{c, \mathrm{DA}}\right)-D_{i, t}+p_{i, t}^{\mathrm{PV}, \mathrm{DA}}$

$: \gamma_{i, t}^{q, \mathrm{DA}} \forall t$

(1de)

$q_{i, t}^{\mathrm{DA}}+q_{i, t, \omega}^{\mathrm{RT}}=\sum_{s}\left(p_{i, s, t}^{d, \mathrm{DA}}+p_{i, s, t, \omega}^{d, \mathrm{RT}}-p_{i, s, t}^{c, \mathrm{DA}}-p_{i, s, t, \omega}^{c, \mathrm{RT}}\right)$

$+p_{i, t, \omega}^{\mathrm{shed}}-D_{i, t}+p_{i, t}^{\mathrm{PV}, \mathrm{DA}}+p_{i, t, \omega}^{\mathrm{PV}, \mathrm{RT}}: \gamma_{i, t, \omega}^{q, \mathrm{RT}} \forall t, \omega$

\}$\forall i \in \mathcal{R}$

where the set of primal variables is given by $\Xi_{\mathcal{R}}=$ $\left\{p_{i, s, t}^{c, \mathrm{DA}}, p_{i, s, t}^{d, \mathrm{DA}}, e_{i, s, t}^{\mathrm{DA}}, p_{i, s, t}^{c, \max , \mathrm{DA}}, p_{i, s, t}^{d, \max , \mathrm{DA}}, e_{i, s, t}^{\max , \mathrm{DA}}, p_{i, s, t, \omega}^{c, \mathrm{RT}}\right.$, $\left.p_{i, s, t, \omega}^{d, \mathrm{RT}}, e_{i, s, t, \omega}^{\mathrm{RT}}, q_{i, t}^{\mathrm{DA}}, q_{i, t, \omega}^{\mathrm{RT}}, p_{i, t, \omega}^{\mathrm{shed}}, p_{i, t}^{\mathrm{PV}, \mathrm{DA}}, p_{i, t, \omega}^{\mathrm{PV}, \mathrm{RT}}\right\}$. The prosumer operates in a similar way as the consumer, but it has access to an additional source of energy coming from its PV installation. Variable $p_{i, t}^{\mathrm{PV}, \mathrm{DA}}$ expresses the utilized PV power in DA and is bounded by its corresponding PV forecast $G_{i, t}^{\mathrm{DA}}$. Variable $p_{i, t, \omega}^{\mathrm{PV}, \mathrm{RT}}$ refers to the adjusted PV power in RT according to each PV scenario realization.

We now provide the profit-maximization problem of a storage owner who supplies PSRs and possesses $s$ units of storage. This problem is given by $1 \mathrm{e}$ :

$$
\begin{aligned}
& \underset{p_{s, t}^{c, \mathrm{ri}, \mathrm{DA}}, p_{s, t}^{d, \mathrm{ri}, \mathrm{DA}}, e_{s, t}^{\mathrm{ri}, \mathrm{DA}}}{\operatorname{Maximize}} \sum_{t, s}\left(p_{s, t}^{c, \mathrm{ri}, \mathrm{DA}} \mu_{s, t}^{c, \mathrm{DA}}+p_{s, t}^{d, \mathrm{ri}, \mathrm{DA}} \mu_{s, t}^{d, \mathrm{DA}}\right. \\
& \left.+e_{s, t}^{\mathrm{ri}, \mathrm{DA}} \mu_{s, t}^{e, \mathrm{DA}}\right)
\end{aligned}
$$

subject to:

$0 \leq p_{s, t}^{c, \mathrm{ri}, \mathrm{DA}} \leq P_{s}^{c, \max }: \underline{\phi}_{s, t}^{c, \mathrm{DA}}, \bar{\phi}_{s, t}^{c, \mathrm{DA}} \forall t, s$ 


$$
\begin{aligned}
& 0 \leq p_{s, t}^{d, \mathrm{ri}, \mathrm{DA}} \leq P_{s}^{d, \max }: \underline{\phi}_{s, t}^{d, \mathrm{DA}}, \bar{\phi}_{s, t}^{d, \mathrm{DA}} \forall t, s \\
& 0 \leq e_{s, t}^{\mathrm{ri}, \mathrm{DA}} \leq E_{s}^{\max }: \underline{\phi}_{s, t}^{e, \mathrm{DA}}, \bar{\phi}_{s, t}^{e, \mathrm{DA}} \forall t, s
\end{aligned}
$$

where $p_{s, t}^{c, \mathrm{ri}, \mathrm{DA}}, p_{s, t}^{d, \mathrm{ri}, \mathrm{DA}}$, and $e_{s, t}^{\mathrm{ri}, \mathrm{DA}}$ are the primal variables expressing the provided charge, discharge, and capacity PSRs, respectively. The storage owner profits from selling the PSRs in DA, as declared by its objective function (1ea). Note that when the storage owner operates as a PSR supplier, its payoff comes exclusively from selling the rights. The provided PSRs are bounded by their corresponding maximum charge $\left(P_{s}^{c, \max }\right)$, discharge $\left(P_{s}^{d, \max }\right)$, and capacity $\left(E_{s}^{\max }\right)$ storage limits.

Lastly, the profit-maximization problem for the grid owner who is indeed a spatial arbitrageur [19] is given by (1f) below:

$$
\begin{aligned}
& \underset{p_{t}^{\text {flow }, \mathrm{DA}}, p_{t, \omega}^{\text {flow, RT }}}{\operatorname{Maximize}} \sum_{t}\left[p_{t}^{\text {flow,DA }}\left(\lambda_{t}^{\mathrm{DA}}-\lambda_{t}^{\mathrm{loc}, \mathrm{DA}}\right)\right. \\
& \left.+\sum_{\omega} \pi_{\omega} p_{t, \omega}^{\mathrm{flow}, \mathrm{RT}}\left(\lambda_{t, \omega}^{\mathrm{RT}}-\frac{\lambda_{t, \omega}^{\mathrm{loc}, \mathrm{RT}}}{\pi_{\omega}}\right)\right]
\end{aligned}
$$

subject to:

$$
\begin{aligned}
& -L \leq p_{t}^{\text {flow,DA }} \leq L: \underline{\beta}_{t}^{\mathrm{DA}}, \bar{\beta}_{t}^{\mathrm{DA}} \forall t \\
& -L \leq p_{t}^{\text {flow,DA }}+p_{t, \omega}^{\text {flow }, \mathrm{RT}} \leq L: \underline{\beta}_{t, \omega}^{\mathrm{RT}}, \bar{\beta}_{t, \omega}^{\mathrm{RT}} \forall t, \omega
\end{aligned}
$$

where parameter $L$ is the capacity of the grid (equivalent to a single line) to connect the local market to the distribution system. Variables $p_{t}^{\text {flow,DA }}$ and $p_{t, \omega}^{\text {flow, RT }}$ denote the power flow through that line (positive if the local market transfers power to the distribution system, negative if otherwise) in DA and $\mathrm{RT}$, respectively. Objective function (1fa maximizes the profit of the grid owner. It performs spatial arbitrage between the distribution-level market and the local market in DA at prices $\lambda_{t}^{\mathrm{DA}}$ and $\lambda_{t}^{\mathrm{loc}, \mathrm{DA}}$, and/or in RT at prices $\lambda_{t, \omega}^{\mathrm{RT}}$ and $\lambda_{t, \omega}^{\mathrm{loc}, \mathrm{RT}}$, respectively. The distribution-level and local market prices are expected to be different when the line is congested. More specifically, for a congested line and positive power flow it is anticipated that $\lambda_{t}^{\text {loc,DA }}<\lambda_{t}^{\mathrm{DA}}$, while for a negative power flow $\lambda_{t}^{\text {loc,DA }}>\lambda_{t}^{\mathrm{DA}}$. The same applies to the RT electricity price.

Finally, the shared linking constraints related to PSRs, as well as the power balance constraint are given by $(1 \mathrm{~g})$ below:

$$
\begin{aligned}
& p_{s, t}^{c, \mathrm{ri}, \mathrm{DA}}=\sum_{i \in(\mathcal{A} \cup \mathcal{P} \cup \mathcal{C} \cup \mathcal{R})} p_{i, s, t}^{c, \max , \mathrm{DA}}: \mu_{s, t}^{c, \mathrm{DA}} \forall s, t \\
& p_{s, t}^{d, \mathrm{ri}, \mathrm{DA}}=\sum_{i \in(\mathcal{A} \cup \mathcal{P} \cup \mathcal{C} \cup \mathcal{R})} p_{i, s, t}^{d, \max , \mathrm{DA}}: \mu_{s, t}^{d, \mathrm{DA}} \forall s, t \\
& e_{s, t}^{\mathrm{ri}, \mathrm{DA}}=\sum_{i \in(\mathcal{A} \cup \mathcal{P} \cup \mathcal{C} \cup \mathcal{R})} e_{i, t}^{\max , \mathrm{DA}}: \mu_{s, t}^{e, \mathrm{DA}} \forall s, t \\
& p_{t}^{\text {flow }, \mathrm{DA}}=\sum_{i \in \mathcal{A}, s}\left(p_{i, s, t}^{d, \mathrm{DA}}-p_{i, s, t}^{c, \mathrm{DA}}\right)+\sum_{i \in(\mathcal{C} \cup \mathcal{R})} q_{i, t}^{\mathrm{DA}} \\
& +\sum_{i \in \mathcal{P}}\left(p_{i, t}^{g, \mathrm{DA}}+\sum_{s} p_{i, s, t}^{d, \mathrm{DA}}\right): \lambda_{t}^{\mathrm{loc}, \mathrm{DA}} \forall t \\
& p_{t, \omega}^{\text {flow }, \mathrm{RT}}=\sum_{i \in \mathcal{A}, s}\left(p_{i, s, t, \omega}^{d, \mathrm{RT}}-p_{i, s, t, \omega}^{c, \mathrm{RT}}\right)+\sum_{i \in(\mathcal{C} \cup \mathcal{R})} q_{i, t, \omega}^{\mathrm{RT}}
\end{aligned}
$$

$$
+\sum_{i \in \mathcal{P}}\left(p_{i, t, \omega}^{g, \mathrm{RT}}+\sum_{s} p_{i, s, t, \omega}^{d, \mathrm{RT}}\right): \lambda_{t, \omega}^{\mathrm{loc}, \mathrm{RT}} \forall t, \omega .
$$

Constraints (1ga)-(1gc), that correspond to charge, discharge and capacity rights respectively, imply that for each storage and in each hour, the total purchased PSRs by all the players should be equal to the total ones sold. Constraint 1gd enforces the power balance in DA, while (1ge imposes such a balance in RT. Note that the shadow prices of (1g) provide the PSR and the DA/RT local energy prices.

The solution of the equilibrium problem can be obtained by simultaneously solving the Karush-Kuhn-Tucker (KKT) conditions of all optimization problems of the players involved together with the shared linking constraints [23]. The resulting models are characterized as mixed-linear complementarity problems (MLCP) and can be solved using the PATH solver [24] or any other MLCP solver. The optimality conditions of the Equilibrium Model (i.e., the collection of the KKT and linking conditions) are provided in the online Appendix [17].

\section{B. Equivalent Optimization Model}

This is a linear optimization model in which the objective is to minimize the local system cost in expectation. The model is given by (2) below:

$$
\begin{aligned}
& \underset{\Xi \mathrm{Opt}-\mathrm{MP}}{\operatorname{Minimize}} \sum_{t}-\lambda_{t}^{\mathrm{DA}} p_{t}^{\mathrm{flow}, \mathrm{DA}}-\sum_{i \in \mathcal{I}, s} \tilde{\lambda} e_{i, s, T}^{\mathrm{DA}} \\
& +\sum_{t, \omega} \pi_{\omega}\left(-\lambda_{t, \omega}^{\mathrm{RT}} p_{t, \omega}^{\mathrm{flow}, \mathrm{RT}}+\sum_{i \in(\mathcal{C} \cup \mathcal{R})} V_{i} p_{i, t, \omega}^{\text {shed }}\right) \\
& -\sum_{i \in \mathcal{I}, s, \omega} \pi_{\omega} \tilde{\lambda} e_{i, s, T, \omega}^{\mathrm{RT}}
\end{aligned}
$$

subject to:

$$
\begin{aligned}
& (1 \mathrm{ab})-1 \mathrm{ak} ; 1 \mathrm{bb}-11 \mathrm{bd} ; 1 \mathrm{cb}-1 \mathrm{ce} ; 1 \mathrm{db}-1 \mathrm{df} ; \\
& 11 \mathrm{eb}-1 \mathrm{ed} ; 1 \mathrm{fb}-1 \mathrm{fc} ; 1 \mathrm{1ga}-1 \mathrm{ge}
\end{aligned}
$$

where $\mathcal{I}=\{\mathcal{A}, \mathcal{P}, \mathcal{C}, \mathcal{R}\}$. The set of decision variables of problem (2a), i.e., $\Xi^{\mathrm{Opt}-\mathrm{MP}}$, includes all variables stated in problems (1a)-11d, (1e) and (1f) of the Equilibrium Model. Furthermore, the set of constraints stated in (2b) contains identical constraints to the ones considered by the players in the Equilibrium Model, as well as the same shared linking constraints.

It is straightforward to verify that the Optimization Model is equivalent to the the Equilibrium Model. That is because the KKTs of Equilibrium Model are identical to the KKTs of Optimization Model, as described in the electronic companion [17]. The advantage of this mathematical proof is the conclusion that instead of the Equilibrium Model, we can solve its equivalent Optimization Model. Contrary to an equilibrium model which its settlement requires the concurrent solution of a set of multiple (and often numerous) linear equations, an optimization model solves a centrally defined operational objective which usually requires less computational resources, and thus it is faster to solve. At the same time, this proof suggests that all desirable market properties that hold in the Equilibrium Model are preserved in the Optimization Model 
as well. However, we need to point out that economic equilibrium models posed as complementarity ones do not always have an equivalent single optimization model, as discussed in [23]. Usually, diverse expressions of market distortion prevent equilibrium models from achieving the minimum social cost or being transformed to equivalent single optimization models. These expressions may include feed-in premium tariffs, market power, risk aversion of players, etc. [25].

\section{Numerical Results}

In this section, we demonstrate the functioning of the proposed PSR auction mechanism in a two-stage market setup through the numerical evaluation of the methods presented earlier. We examine how the storage owner's payoff is affected depending on its participation in the market (as arbitrageur or as PSR provider) and we also assess the effect of this decision on system's outcomes and each player's results. We also examine each market participant's financial performance and their individual interaction with the PSR provider in terms of exchanged rights. To perform this kind of analysis and for tractability purposes, we have selected a limited number of representative market participants in the case studies. In reality, however, the market would consist of many more participants and scale to more agents.

We consider three different cases in total. Case 1 consists of eight participants: two wind producers, two consumers, two prosumers, the storage owner, and the grid owner. Specifically, in Case 1 the ES owner uses the storage at its own interest participating in both DA and RT markets (as the rest of the market participants). One can easily apprehend that in such a format, the rest of the market participants have no access to ES resources. Case 2 corresponds to the Optimization Model and consists of the same participants as in Case 1 with the addition of an extra participant, the arbitrageur (owning though no physical asset itself). In this case, the participants (apart from the grid owner) compete for the PSRs provided exclusively by the storage owner in DA market. Therefore, the ES owner participates only in DA market and its revenue comes exclusively from selling the PSRs. The obtained results are compared with those of a benchmark, called Base Case. In this case, storage (in either form) is entirely excluded from the system. In all cases, a positive payoff indicates profit, while a negative one implies the cost for a market participant.

In all cases, the total available (dis)charge and capacity nominal values remain constant for each storage type. We have assumed two different storage types, type 1 and type 2 , with type 2 having better (dis)charge efficiencies. All the cases have been modeled assuming zero initial SOC for the ES. As input data, Fig. 1 depicts the DA forecasts for wind and PV power production, as well as for the distribution-level DA energy price $\lambda_{t}^{\mathrm{DA}}$ for the next 24 hours. The distribution-level DA price reflects the hourly wholesale market price [26]. All the rest technical details and input data (storage capacities, efficiencies, RT scenarios, etc.) related to the case studies are provided in Appendix C. In particular, we consider 40 scenarios for in-sample analysis, and 365 scenarios for ex-post out-of-sample verification. All linear optimization problems are solved by CPLEX/GAMS using a usual desktop, and



Fig. 1. DA forecasts for wind-PV power production and energy price

CPU time for different case studies varies between 1 and 147 seconds.

The main criterion with which the case studies evaluate the local market model is the total expected system cost (TESC), as given in Table I It is interesting to note that the participation of ES, either as regular market player (Case 1) or as PSR provider (Case 2), decreases the total expected system cost. One might also note that the TESC for Cases 1 and 2 are equal. It is also of interest that the expected payoff of each individual participant in both Cases 1 and 2 is identical (see Table III.

TABLE I

TOTAL EXPECTEd SYSTEM Cost [\$] FOR ALl CASES

\begin{tabular}{c|c|c}
\hline Base (no ES) & 1 (ES as arbitrageur) & 2 (ES as PSR provider) \\
\hline 98.81 & 90.39 & 90.39 \\
\hline
\end{tabular}

This is mainly due to the unlimited access to flexibility the distribution system grants to the local market participants, even if it is acquired at a premium price (i.e., when the line is congested). The benefits of local flexibility which is provided by the access to storage facilities through the concept of PSRs, would be more profound to the economic results of the market participants if a limitation to the flexibility offered by the distribution system was established. In addition, although the expected payoff of each market participant is the same across the cases, the volatility of its payoff over scenarios in the two cases is different.

Fig. 2 breaks down the total expected payoff of a number of players to their payoff in DA and RT. It also illustrates the 10th and 90th percentile of payoffs in RT. Several observations emerge from the comparison of the case studies. Starting with the storage owner (SO), one may notice that the profit (\$8.33) of the storage owner as a PSR provider (coming exclusively from DA market in Case 2) is equal to its expected profit ( $\$ 7.43$ in DA plus $\$ 15.76$ in RT, Case 1) as a regular market participant. However, contrary to the former, completely volatility-free option, the latter engages some uncertainty coming from the RT that involves renewables and price volatility. For example, the 10th percentile of ES owner in Fig. 2 indicates that in $10 \%$ of all possible 
TABLE II

Expected Payoff of Each Market Participant Across All Case STUDIES [\$]

\begin{tabular}{c|c|c|c|c|c|c|c|c|c}
\hline Case & WP1 & WP2 & CON1 & CON2 & PRO1 & PRO2 & SO & ARB & GO \\
\hline Base & 51.99 & 42.6 & -52.58 & -46.69 & -39.38 & -54.76 & N/A & N/A & 0 \\
\hline 1 & 51.99 & 42.6 & -52.63 & -46.73 & -39.42 & -54.83 & 8.33 & N/A & 0.29 \\
\hline 2 & 51.99 & 42.6 & -52.63 & -46.73 & -39.42 & -54.83 & 8.33 & 0 & 0.29 \\
\hline
\end{tabular}

[WP stands for wind producer, CON for consumer, PRO for prosumer, SO for storage owner, ARB arbitrageur and GO for grid owner.
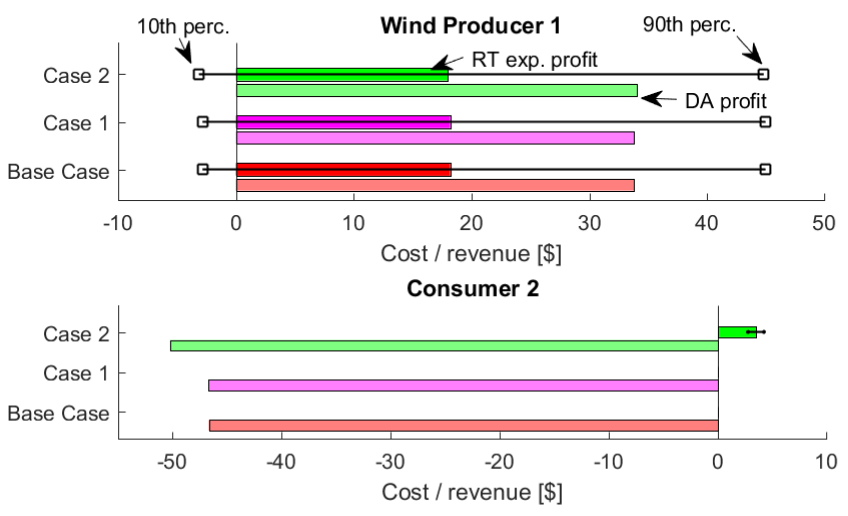

Prosumer 1
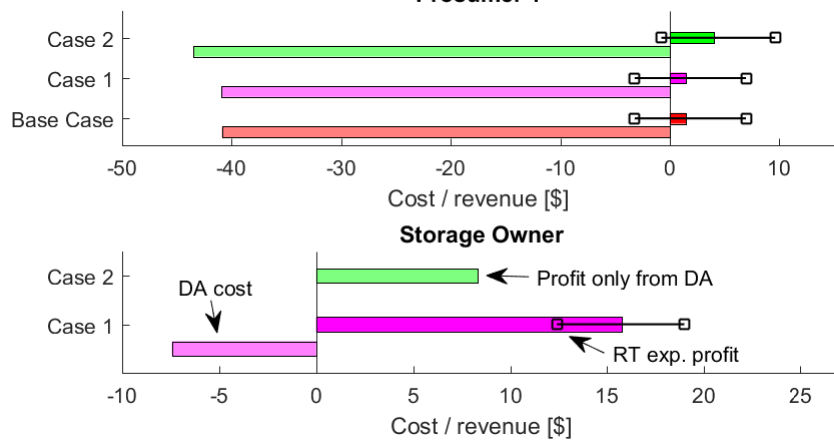

Fig. 2. Players' individual expected profit/cost in Cases 1, 2 and Base Case [\$]

scenario realizations, the storage owner's RT revenue is less than $\$ 12.4$ resulting in a significantly smaller total expected profit compared to its certain profit from DA only in Case 2. Considering that a player with conservative preferences is willing to accept a profit smaller than the expected value but with greater certainty, the PSR format is ideal for such a player, not only because it removes all uncertainty associated with RT, but it also delivers a total revenue upfront which is equal to the expected one. This result is significant as it suggests that the PSR format is more attractive for storage owners who are reluctant to take risks associated with low profit due to the potential realization of undesirable scenarios in RT. On the other hand, a storage owner who requires a profit greater than the expected value, would choose to act as a regular market player, anticipating RT revenue higher than the 50th percentile. Finally, less conservative storage owners who simply want to maximize their expected value would be indifferent, since both PSR and RT market setups would deliver the same results.

Profit volatility does not disappear from the market, it rather passes from the storage owner to a certain category of market participants within the PSR format. This is reflected on the results illustrated in Table $\Pi$ and Fig. 2 According to
Table III the players' total revenue (excluding storage owner) is only slightly modified along the case studies. However, for a specific group of market participants (consumers and prosumers), we observe an increase in their DA cost which is offset by anticipating a higher RT profit. For instance, while in Base Case and Case 1 both consumers participate in DA market only, in Case 2 they also get involved in RT market. Similarly, the prosumers increase their involvement in RT market under the PSR format, a fact that is expressed by their raised expected profit in RT. A common remark for both consumers and prosumers is that their increased exposure in RT is accompanied with a concurrent increase in their DA cost. Further analysis of the results suggests that this increase in DA cost for the players, is mainly due to the acquisition of ES resources through the PSRs (which are only traded in DA) aiming to improve their RT expected cost, either by arbitraging or by exploiting the ES residual energy. However, not all market participants are affected by the format that storage participates in the market. The wind producers are rather indifferent and behave similarly in all cases, regardless the manner ES takes part in the market. Finally, the grid owner reports a very small profit in Cases 1 and 2 and only in DA, indicating that in RT the line is never congested.
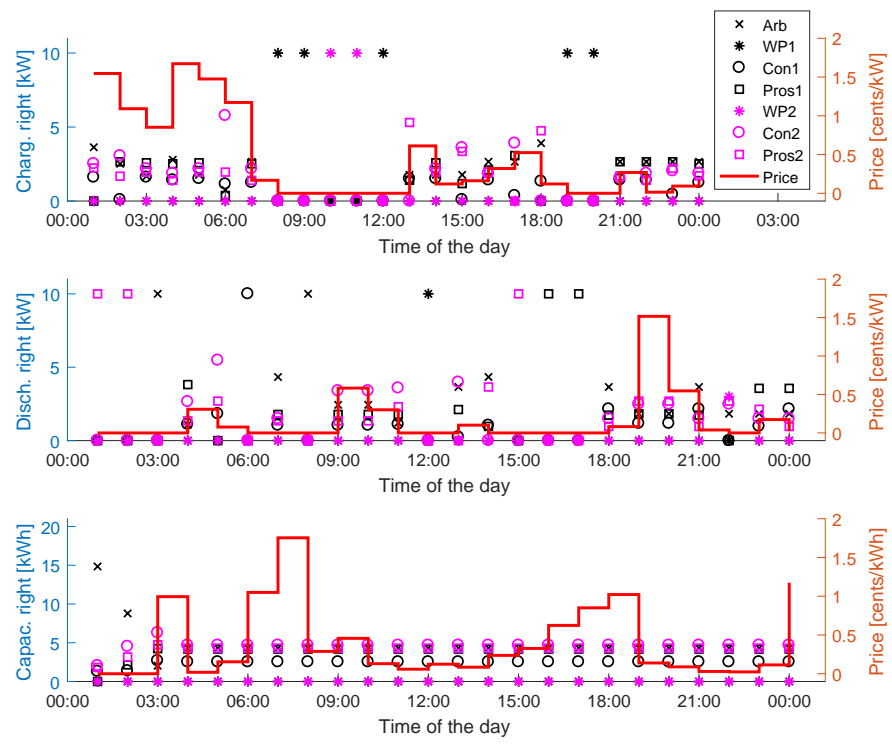

Fig. 3. Individual acquisition of charge rights (upper plot), discharge rights (intermediate plot), capacity rights (lower plot) for market participants and their clearing prices (ES type 1)

Figs. 3 and 4 show the PSRs acquired by the market participants' in a daily auction with the hourly market-clearing prices for both types of storage offered. The price of an hourly allocated auctioned right $\left(\mu_{s, t}^{c, \mathrm{DA}}, \mu_{s, t}^{d, \mathrm{DA}}, \mu_{s, t}^{e, \mathrm{DA}}\right)$ only takes a non-zero value when the inequalities (1eb)-1ed are binding. A non-zero price reflects the willingness-to-pay as expressed by the demand bid of the marginally cleared player for a specific PSR. One may notice a similar pattern in the demand and the price of PSRs for both storage types. The charge rights are mainly granted during the morning hours leading to an increased provision of capacity rights for the same period. This happens because it is essential for players to 

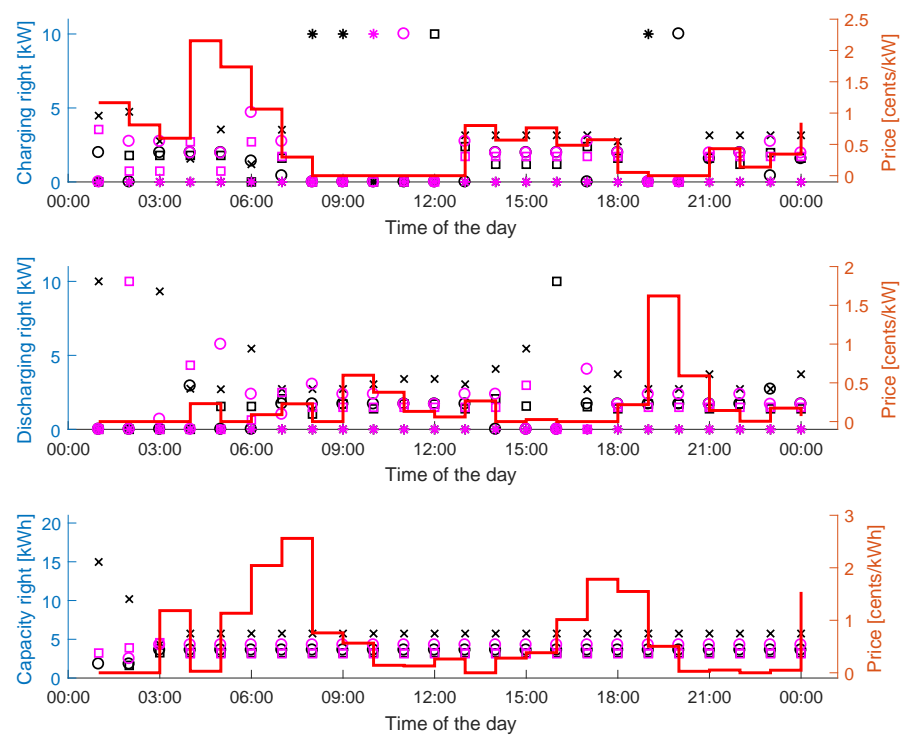

Fig. 4. Local market outcomes (as in Fig. 3 for ES type 2

maintain the ownership of the ES resources (through capacity rights) they have already charged, until they decide to fully discharge it; otherwise they might not be able to gain from their stored energy. The discharge rights are in higher demand mainly during the hours that it is more favourable to sell the energy back into the grid based on the local price scheme. As expected, the price for the capacity rights surge again during the time periods prior discharging, as the players try to secure their stored energy until they profit from it. Finally, the increased provision of charge and capacity rights during the late hours of the day is due to the players' intention to profit from the value of residual energy at the end of daily horizon.

The findings regarding the PSR auction (as also illustrated in Figs. 3 and 4 provide us with two remarks. First, during the time periods of a day where competition is low, PSRs can be granted at zero price. Second, as we expected, the higher dis(charge) efficiencies of ES type 2 result in higher PSR prices for this type of storage.

To demonstrate the effectiveness of the stochastic optimization approach, we perform additional out-of-sample simulations. Specifically, the RT market is cleared for 365 daily data vectors (including renewable generation values and RT prices), while the DA decisions are fixed to those obtained from stochastic optimization. Then, we compute the actual system cost that consists of the fixed DA system cost plus the expected system cost obtained from the deterministic out-of-sample simulations. The individual market participants' revenue is calculated in a similar way. One should note that, contrary to the stochastic optimization approach where all expected RT results are calculated based on the probability-weighted scenarios, in the out-of-sample approach we calculate the RT results assigning a probability equal to $\frac{1}{365}$ to each of the 365 simulations. Table III shows the percentage change of the total average system cost and the individual players' revenue in the out-of-sample approach compared to the stochastic optimization one for all case studies.
TABLE III

Percentage Change in Out-OF-SAmple Simulations for Local SySTEM COST AND INDIVIDUAL Player REVENUE COMPARED TO IN-SAMPLE ANALYSIS [\%]

\begin{tabular}{c|c|c|c|c|c|c|c|c|c|c}
\hline Case & TESC & WP1 & WP2 & CON1 & CON2 & PRO1 & PRO2 & SO & ARB & GO \\
\hline Base & -4.43 & -1.02 & 7.89 & 0 & 0 & 2.65 & 0.95 & N/A & N/A & 0 \\
\hline 1 & -3.15 & -1.02 & 7.90 & 0 & 0 & 2.65 & 0.95 & $\mathbf{- 1 8 . 4 1}$ & N/A & 0 \\
\hline 2 & -3.02 & -1.01 & 7.89 & -0.41 & -0.89 & 1.97 & 0.28 & $\mathbf{0}$ & INF & 0 \\
\hline
\end{tabular}

The out-of-sample findings need to be interpreted with caution and within the context they are obtained. First, aligned with the in-sample analysis, the introduction of storage in the system in either form decreases the total average system cost, as seen in Table III. However, the revenues of market participants seem to be affected in a diverse way. For example, the profit for WP1 is lower in the out-of-sample approach, while the profit of WP2 is comparatively increased. This is mainly due to the impact of each one's DA decisions in relation with the possible RT outcomes. It also shows that WP2 had a relatively unpropitious DA forecast compared to the actual RT realizations. In addition, we notice a zero alteration in consumers' cost for Base Case and Case 1. The reason is that, like in stochastic programming approach, in out-ofsample setup the consumers do not participate in RT market in these two cases. Therefore, their cost remained the same, expressed only by the DA fixed decisions. The grid owner's profit also comes from DA only, showing thus zero change in out-of-sample simulations.

The storage owner on the other hand, is among the market participants with the heaviest financial losses within a specific market format (Case 1). Indeed, the total expected profit of the SO is almost $20 \%$ less in the out-of-sample setup compared to the stochastic programming approach ( $\$ 6.79$ instead of \$8.33). On the contrary, since the profit of the SO in the PSR format (Case 2) comes exclusively from the DA market, the out-of-sample simulations (with which only the RT market is cleared) leave its revenue unaffected. These results indicate the potential risk involved when the SO acts as a regular market player and not as a PSR provider. However, one must take into account that the findings in this work are excessively dependant on the considered scenarios for RT in stochastic programming and also on the quality of DA forecasts each player has. Finally, as occurred with the case of the SO, the out-of-sample simulations resulted in a final negative profit for the arbitrageur.

Coupled with the PSRs, passive storage extends the process through which ES owners profit: instead of directly arbitraging temporal price variations, they profit through the provision of physical storage rights, whose value depends on price variations. Storage owners who are reluctant in taking risk can beneficially enter this process and hedge against RT price volatility. On the other hand, risk-seeking market participants gain access to ES resources through the PSRs anticipating a higher expected profit in RT market. The aforementioned remarks are also supported by the conducted out-of-sample analysis. Our proposed model through the suggested auction design, generates PSR prices that are intuitively clear and transparent. In addition, these prices are equilibrium- 
supporting in the sense that each PSR holder would want to follow the (dis)charge actions specified by the PSR allocation.

\section{CONCLUSION}

This paper proposes a local market design where the PSRs are allocated for a period of 24 hours based on periodical (daily) market clearing auctions. We first prove that the initial Nash equilibrium model can be substituted with its equivalent optimization one ensuring the same desirable economic properties in the market. The potential added value of the ES to the system is numerically evaluated by our models and their computational applications. Furthermore, we examine two different market formulations under which a storage asset could participate in DA and RT markets. We find out that the certain profit of a storage owner in DA when acting as a PSR provider is equal to its expected profit as a regular market participant providing a risk-free option with the same economic return. At the same time, the PSRs grant access to the rest market players who can exploit it at their best interest. Additional out-of-sample simulations are in line with the results obtained from stochastic programming.

Future work will concentrate on the introduction of a risk measure (e.g., the conditional value-at-risk) to quantify the average loss for the market participants over a specified time period of unlikely renewables and price scenarios.

\section{APPENDIX A: NOMENCLATURE}

\section{A. Indices:}

$i \quad$ Index for all market players

$s \quad$ Index for storage units

$t \quad$ Index for operating hours

$\omega \quad$ Index for renewable generation and electricity price scenarios in real-time market

\section{B. Sets}

$\begin{array}{ll}\mathcal{A} & \text { Set of arbitrageurs with no physical storage asset } \\ \mathcal{C} & \text { Set of consumers with no physical storage asset } \\ \mathcal{P} & \text { Set of wind producers with no physical storage } \\ & \text { asset } \\ \mathcal{R} & \text { Set of prosumers with no physical storage asset }\end{array}$

\section{Parameters}

$D_{i, t} \quad$ Load demand of player $i$ in hour $t[\mathrm{~kW}]$

$E_{s}^{\max } \quad$ Nominal capacity of storage $s[\mathrm{kWh}]$

$E_{i, s}^{\text {ini }} \quad$ Initial energy of storage $s$ accessed by player $i$ [kWh]

$G_{i, t}^{\mathrm{DA}} \quad$ Maximum renewable production of player $i$ in hour $t$ that can be scheduled at the scheduling stage $[\mathrm{kW}]$

$G_{i, t, \omega}^{\mathrm{RT}} \quad$ Renewable production realization of player $i$ in hour $t$ under scenario $\omega[\mathrm{kW}]$

$L \quad$ Line capacity $[\mathrm{kW}]$

$P_{s}^{c, \max } \quad$ Maximum charge rate of storage $s[\mathrm{~kW}]$

$P_{s}^{d, \max } \quad$ Maximum discharge rate of storage $s[\mathrm{~kW}]$

$V_{i} \quad$ Value of lost load for player $i[\$ / \mathrm{kWh}]$

$\tilde{\lambda} \quad$ Value of storage residual energy $[\$ / \mathrm{kWh}]$

$\lambda_{t}^{\mathrm{DA}} \quad$ Forecast in day-ahead market for distributionlevel electricity price in hour $t[\$ / \mathrm{kWh}]$

$\lambda_{t, \omega}^{\mathrm{RT}} \quad$ Realization in real-time market for distributionlevel electricity price in hour $t$ under scenario $\omega$ $[\$ / \mathrm{kWh}]$

$\pi_{\omega} \quad$ Probability of scenario $\omega$

\section{Primal variables (day-ahead market):}

$e_{i, s, t}^{\mathrm{DA}} \quad$ Stored energy of storage $s$ accessed by player $i$ in time $t[\mathrm{kWh}]$

$e_{i, s, t}^{\max , \mathrm{DA}} \quad$ Maximum capacity right of storage $s$ owned by ri,DA player $i$ in time $t[\mathrm{kWh}]$

$e_{s, t}^{\text {ri,DA }} \quad$ Total capacity right offered from storage $s$ in time $t[\mathrm{kWh}]$

$p_{i, s, t}^{c, \mathrm{DA}} \quad$ Charge power of storage $s$ accessed by player $i$ in time $t[\mathrm{~kW}]$

$p_{i, s, t}^{c, \text { max,DA }}$ Maximum charge right of storage $s$ owned by player $i$ in time $t[\mathrm{~kW}]$

$p_{s, t}^{c, \text { ri,DA }} \quad$ Total charge right offered from storage $s$ in time $t[\mathrm{~kW}]$

$p_{i, s, t}^{d, \mathrm{DA}} \quad$ Discharge power of storage $s$ accessed by player $i$ in time $t[\mathrm{~kW}]$

$p_{i, s, t}^{d, \max , \mathrm{DA}}$ Maximum discharge right of storage $s$ owned by player $i$ in time $t[\mathrm{~kW}]$

$p_{s, t}^{d, \mathrm{ri}, \mathrm{DA}} \quad$ Total discharge right offered from storage $s$ in time $t[\mathrm{~kW}]$

$p_{i, t}^{g, \mathrm{DA}} \quad$ Power from wind producer $i$ directly sold to the grid in time $t[\mathrm{~kW}]$

$p_{i, t}^{\mathrm{PV}, \mathrm{DA}} \quad$ Photovoltaic power utilized from prosumer $i$ in time $t[\mathrm{~kW}]$

$p_{t}^{\text {flow,DA }}$ Total power flow through distribution line in time $t[\mathrm{~kW}]$

$q_{i, t}^{\mathrm{DA}} \quad$ Net power consumption/generation of player $i$ in time $t[\mathrm{~kW}]$

\section{E. Primal variables (real-time market):}

$e_{i, s, t, \omega}^{\mathrm{RT}}$

$p_{i, s, t, \omega}^{c, \mathrm{RT}}$

$p_{i, s, t, \omega}^{d, \mathrm{RT}}$

$p_{i, t, \omega}^{g, \mathrm{RT}}$

$p_{i, t, \mathrm{PV}}^{\mathrm{PV}}$

$p_{i, t, \omega}^{\mathrm{PV}}$

$p_{t, \omega}^{\text {flow, RT }}$

$p_{i, t, \omega}^{\text {shed }}$

$q_{i, t, \omega}^{\mathrm{RT}}$
Stored energy adjustment of storage $s$ accessed by player $i$ in time $t$ under scenario $\omega$ [kWh]

Charge power adjustment of storage $s$ accessed by player $i$ in time $t$ under scenario $\omega[\mathrm{kW}]$

Discharge power adjustment of storage $s$ accessed by player $i$ in time $t$ under scenario $\omega$ $[\mathrm{kW}]$

Power adjustment from wind producer $i$ directly sold to the grid in time $t$ under scenario $\omega[\mathrm{kW}]$ Photovoltaic power adjustment utilized from prosumer $i$ in time $t$ under scenario $\omega[\mathrm{kW}]$

Total power flow adjustment through distribution line in time $t$ under scenario $\omega[\mathrm{kW}]$

Shed power of player $i$ in time $t$ under scenario $\omega[\mathrm{kW}]$

Net power consumption/generation adjustment of player $i$ in time $t$ under scenario $\omega[\mathrm{kW}]$

\section{F. Dual variables:}

$\mu_{s, t}^{c, \mathrm{DA}}$

Day-ahead price of charge right offered by storage $s$ in time $t[\$ / \mathrm{kW}]$ 


$$
\begin{aligned}
& \mu_{s, t}^{d, \mathrm{DA}} \quad \text { Day-ahead price of discharge right offered by } \\
& \text { storage } s \text { in time } t[\$ / \mathrm{kW}] \\
& \mu_{s, t}^{e, \mathrm{DA}} \quad \text { Day-ahead price of capacity right offered by } \\
& \text { storage } s \text { in time } t[\$ / \mathrm{kWh}] \\
& \lambda_{t}^{\text {loc,DA }} \quad \text { Local market day-ahead price in time } t[\$ / \mathrm{kWh}] \\
& \lambda_{t, \omega}^{\text {loc, RT Probability-weighted local market real-time }} \\
& \text { price in time } t \text { under scenario } \omega[\$ / \mathrm{kWh}]
\end{aligned}
$$

\section{APPEndix B: ReVEnUe AdEQUACy}

This Appendix proves that the Equilibrium Model is revenue-adequate. To this purpose, at the optimal solution, we multiply each expression within the physical storage rights equalities (1ga)-(1gc) by $\mu_{s, t}^{c, \mathrm{DA}}, \mu_{s, t}^{d, \mathrm{DA}}$, and $\mu_{s, t}^{e, \mathrm{DA}}$, respectively. Similarly, all expressions within the power flow equalities $1 \mathrm{gd}$ and $1 \mathrm{ge}$ are multiplied by $\lambda_{t}^{\mathrm{loc}, \mathrm{DA}}$ and $\lambda_{t, \omega}^{\mathrm{loc}, \mathrm{RT}}$ at the optimal solution, respectively. Afterwards, we sum all the obtained equalities, i.e.

$$
\begin{aligned}
& \sum_{s}\left(p_{s, t}^{c, \mathrm{ri}, \mathrm{DA} *} \mu_{s, t}^{c, \mathrm{DA} *}+p_{s, t}^{d, \mathrm{ri}, \mathrm{DA} *} \mu_{s, t}^{d, \mathrm{DA} *}+e_{s, t}^{\mathrm{ri}, \mathrm{DA} *} \mu_{s, t}^{e, \mathrm{DA} *}\right) \\
& +p_{t}^{\text {flow }, \mathrm{DA} *} \lambda_{t}^{\text {loc, } \mathrm{DA} *}+\sum_{\omega} p_{t, \omega}^{\text {flow, } \mathrm{RT} *} \lambda_{t, \omega}^{\text {loc, } \mathrm{RT} *}= \\
& \sum_{i \in \mathcal{I}, s}\left(p_{i, s, t}^{c, \max , \mathrm{DA} *} \mu_{s, t}^{c, \mathrm{DA} *}+p_{i, s, t}^{d, \max , \mathrm{DA} *} \mu_{s, t}^{d, \mathrm{DA} *}+\right. \\
& \left.e_{i, s, t}^{\max , \mathrm{DA} *} \mu_{s, t}^{e, \mathrm{DA} *}\right)+\sum_{i \in \mathcal{A}, s}\left[\left(p_{i, s, t}^{d, \mathrm{DA} *}-p_{i, s, t}^{c, \mathrm{DA} *}\right) \lambda_{t}^{\mathrm{loc}, \mathrm{DA} *}\right]+ \\
& \sum_{i \in \mathcal{P}}\left[\left(p_{i, t}^{g, \mathrm{DA} *}+\sum_{s} p_{i, s, t}^{d, \mathrm{DA} *}\right) \lambda_{t}^{\mathrm{loc}, \mathrm{DA} *}\right]+\sum_{i \in(\mathcal{C} \cup \mathcal{R})} q_{i, t}^{\mathrm{DA} *} \lambda_{t}^{\mathrm{loc}, \mathrm{DA} *} \\
& +\sum_{i \in \mathcal{A}, s, \omega}\left[\left(p_{i, s, t, \omega}^{d, \mathrm{RT} *}-p_{i, s, t, \omega}^{c, \mathrm{RT} *}\right) \lambda_{t, \omega}^{\mathrm{loc}, \mathrm{RT} *}\right]+\sum_{i \in(\mathcal{C} \cup \mathcal{R}), \omega} q_{i, t, \omega}^{\mathrm{DA} *} \lambda_{t, \omega}^{\mathrm{loc}, \mathrm{RT} *} \\
& +\sum_{i \in \mathcal{P}, \omega}\left[\left(p_{i, t, \omega}^{g, \mathrm{RT} *}+\sum_{s} p_{i, s, t, \omega}^{d, \mathrm{RT} *}\right) \lambda_{t, \omega}^{\mathrm{loc}, \mathrm{RT} *}\right] \forall t
\end{aligned}
$$

where superscript $*$ stands for the optimal values.

According to (3), the total payment of the players for their obtained physical storage rights and the their purchased energy to the market operator for DA and RT, i.e., the right-hand side, equals to the total payment of the market operator to the physical storage rights supplier and to the grid owner. Therefore, the market operator never incurs a financial deficit, i.e., the market is revenue-adequate. Similarly, we can prove the revenue-adequacy for Optimization Model.

\section{Appendix C: Parameters and Technical Data For THE CASE STUdies}

We provide here the technical data and the parameter values used in the case studies in Section IV of the main paper. Table IV provides the storage (dis)charge efficiencies, the nominal (dis)charge and capacity rates, the value of lost load, as well as the remaining parameters used in the case studies.

Fig. 5 illustrates the 40 scenarios for production (wind and PV) and energy price (RT), which were generated from historical time series. Specifically, the PV production is a
TABLE IV

PARAMETER VALUes

\begin{tabular}{l|c}
\hline Parameter & Value \\
\hline Charge efficiency storage type $1 \eta_{s}^{c}$ & 0.81 \\
\hline Discharge efficiency storage type $1 \eta_{s}^{d}$ & 0.85 \\
\hline Charge efficiency storage type $2 \eta_{s}^{c}$ & 0.91 \\
\hline Discharge efficiency storage type $2 \eta_{s}^{d}$ & 0.95 \\
\hline Nominal dis(charge) rate (same for type 1 and 2) $P_{s}^{c, \text { max }}, P_{s}^{d, \text { max }}[\mathrm{kW}]$ & 10 \\
\hline Nominal capacity (same for type 1 and 2$) E_{s}^{\max }[\mathrm{kWh}]$ & 20 \\
\hline Grid line capacity $L[\mathrm{~kW}]$ & 59 \\
\hline Value of lost load $V_{i}[\$ / \mathrm{kWh}]$ & 4 \\
\hline Value of storage residual energy $\tilde{\lambda}[\$ / \mathrm{kWh}]$ & 0.28 \\
\hline
\end{tabular}

source of uncertainty related with the prosumers. One should notice that the scenarios are not equiprobable but probabilityweighted. The scenarios and their probabilities form a discrete approximation of the probability distribution of the data process.

In this work, we used a scenario reduction technique based on the study of Growe-Kuska et al. [27]. Specifically, we used this technique to aggregate all five sources of uncertainty into one. That is, a discrete probability has been assigned to each one of the 40 scenarios. Every scenario comprises five 24-hour vectors where each vector corresponds to a specific profile (two vector profiles for wind, two for PV production, and one for the price).

The data used for the out-of-sample simulations are presented in Fig. 6. Specifically, the RT market is cleared for each one of the 365 data vectors (including renewable generation values and RT prices) keeping fixed the DA values obtained from the in-sample analysis in stochastic optimization.

\section{ACKNOWLEDGEMENT}

The authors would like to thank Lesia Mitridati (Georgia Tech), Thomas Baroche (ENS Rennes) and Niklas Vespermann (TU Munich) for their valuable suggestions on the final version of the manuscript. We also thank the three anonymous reviewers for their valuable comments.

\section{REFERENCES}

[1] S. P. Forrester, A. Zaman, J. L. Mathieu and J. X. Johnson, "Policy and market barriers to energy storage providing multiple services," Elect. J., vol. 30, no. 9, pp. 50-56, 2017.

[2] D. Krishnamurthy, C. Uckun, Z. Zhou, P. Thimmapuram, and A Botterud, "Energy storage arbitrage under day-ahead and real-time price uncertainty," IEEE Trans. Power Syst., vol. 33, no. 1, pp. 84-93, 2018.

[3] J. Mitra, "Reliability-based sizing of backup storage," IEEE Trans. Power Syst., vol. 25, no. 2, pp. 1198-1199, 2010

[4] S. Wen, H. Lan, Q. Fu, D. C. Yu, and L. Zhang, "Economic allocation for energy storage system considering wind power distribution," IEEE Trans. Power Syst., vol. 30, no. 2, pp. 644-652, 2015.

[5] M. Ammar and G. Joós, "A short-term energy storage system for voltage quality improvement in distributed wind power," IEEE Trans. Energy Convers., vol. 29, no. 4, pp. 997-1007, 2014.

[6] E. Nasrolahpour, J. Kazempour, H. Zareipour, and W. D. Rosehart, "A bilevel model for participation of a storage system in energy and reserve markets," IEEE Trans. Sustain. Energy, vol. 9, no. 2, pp. 582-598, 2018.

[7] M. Dicorato, G. Forte, M. Pisani, and M. Trovato, "Planning and operating combined wind-storage system in electricity market," IEEE Trans. Sustain. Energy, vol. 3, no. 2, pp. 209-217, 2012.

[8] J. Arteaga and H. Zareipour, "A price-maker/price-taker model for the operation of battery storage systems in electricity markets," IEEE Trans. Smart Grid, vol. 10, no. 6, pp. 6912-6920, 2019. 

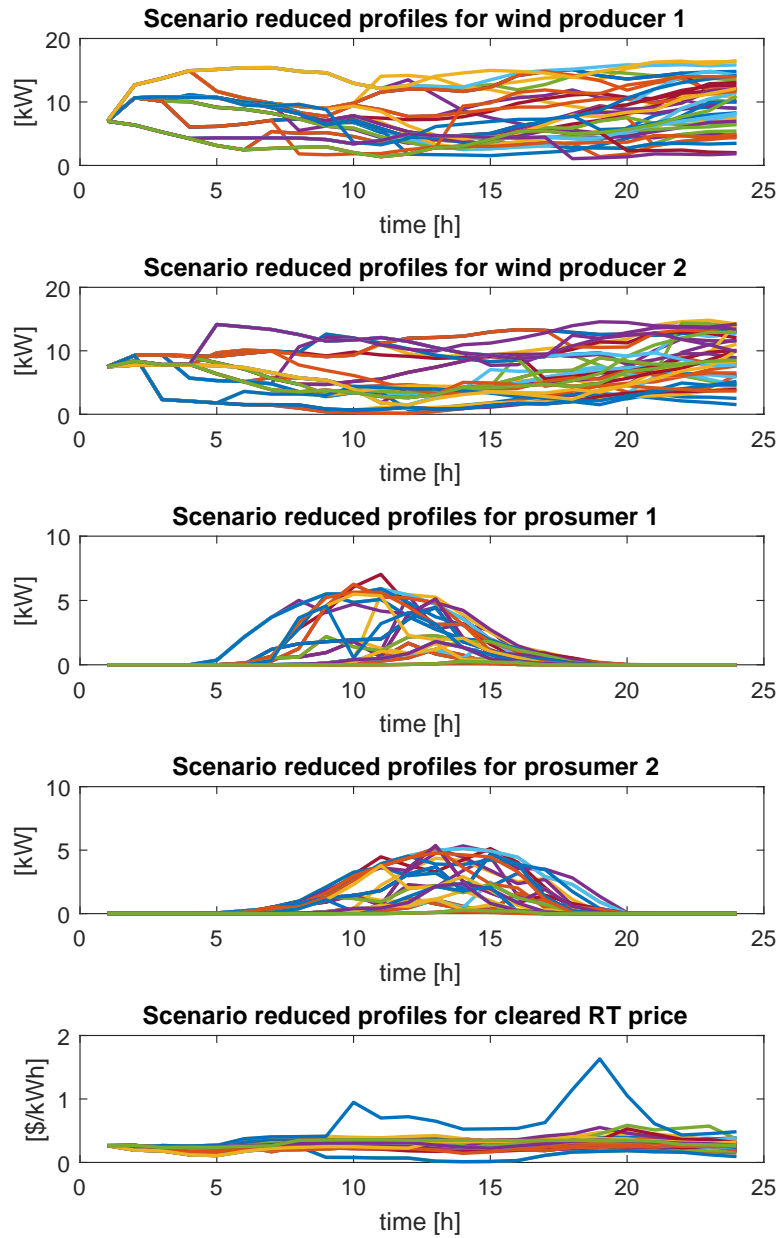

Fig. 5. The 40 scenarios for wind-PV power production and energy price used in the in-sample analysis

[9] E. Nasrolahpour, J. Kazempour, H. Zareipour, and W. D. Rosehart, "Strategic sizing of energy storage facilities in electricity markets," IEEE Trans. Sustain. Energy, vol. 7, no. 4, pp. 1462-1472, 2016.

[10] M. Rahimiyan and L. Baringo, "Strategic Bidding for a virtual power plant in the day-ahead and real-time markets: A price-taker robust optimization approach," IEEE Trans. Power Syst., vol. 31, no. 4, pp. 2676-2687, 2016.

[11] Y. Parag and B. K. Sovacool, "Electricity market design for the prosumer era," Nature Energy, vol. 1, no. 4 pp. 16032, 2016.

[12] J. A. Taylor, "Financial storage rights," IEEE Trans. Power Syst., vol. 30, no. 2, pp. 997-1005, 2015.

[13] D. Muñoz-Álvarez and E. Bitar, "Financial storage rights in electric power networks," J. Reg. Econ., vol. 52, no. 1, pp. 1-23, 2017.

[14] X. He, E. Delarue, W. D'haeseleer, and J.-M. Glachant, "A novel business model for aggregating the values of electricity storage," Energy Policy, vol. 39, no. 3, pp. 1575-1585, 2011.

[15] T. Brijs, D. Huppmann, S. Siddiqui, and R. Belmans, "Auction-based allocation of shared electricity storage resources through physical storage rights," Journal of Energy Storage, vol. 7, pp. 82-92, 2016.

[16] R. Sioshansi, "Using storage-capacity rights to overcome the costrecovery hurdle for energy storage," IEEE Trans. Power Syst., vol. 32, no. 3, pp. 2028-2040, 2017.

[17] D. Thomas, J. Kazempour, A. Papakonstantinou, P. Pinson, O. Deblecker, and C. S. Ioakimidis, "Electronic companion - A local market mechanism for physical storage rights," Available: https://doi.org/10. 5281/zenodo.3305782

[18] F. Moret and P. Pinson, "Energy collectives: a community and fairness based approach to future electricity markets," IEEE Trans. Power Syst., vol. 34, no. 5, pp. 3994-4004, 2019.
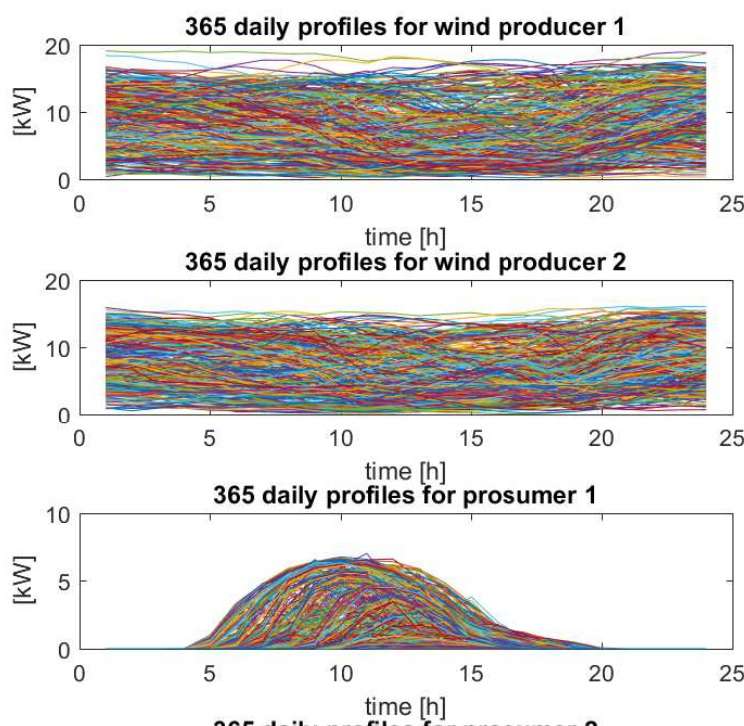

365 daily profiles for prosumer 2
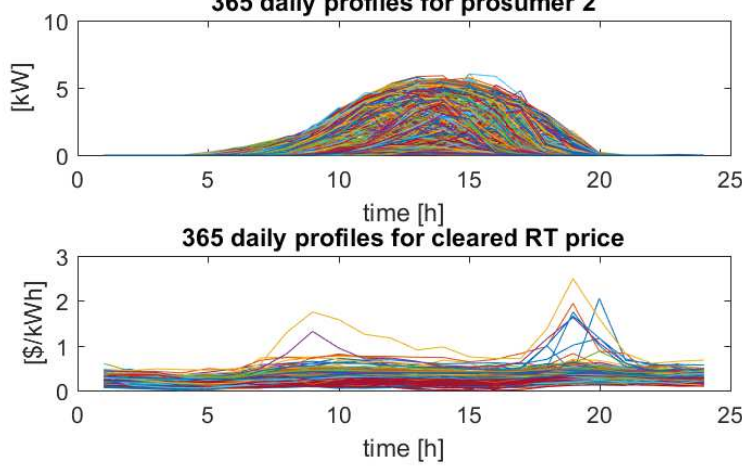

Fig. 6. The 365 daily profiles used in the out-of-sample simulations

[19] J. Kazempour and B. F. Hobbs, "Value of flexible resources, virtual bidding, and self-scheduling in two-settlement electricity markets with wind generation-Part I: principles and competitive model," IEEE Trans. Power Syst., vol. 33, no. 1, pp. 749-759, 2018.

[20] D. Kalathil, C. Wu, K. Poolla, and P. Varaiya, "The sharing economy for the electricity storage," IEEE Trans. Smart Grid, vol. 10, no. 1, pp. 556-567, 2019.

[21] J. M. Morales, A. J. Conejo, K. Liu, and J. Zhong, "Pricing electricity in pools with wind producers," IEEE Trans. Power Syst., vol. 27, no. 3, pp. 1366-1376, 2012.

[22] H. Ding, P. Pinson, Z. Hu, and Y. Song, "Optimal offering and operating strategies for wind-storage systems with linear decision rules," IEEE Trans. Power Syst., vol. 31, no. 6, pp. 4755-4764, 2016.

[23] S. A. Gabriel, A. J. Conejo, J. D. Fuller, B. F. Hobbs, and C. Ruiz, Complementarity modeling in energy markets. NY, Springer, 2012.

[24] M. C. Ferris and T. S. Munson, "Complementarity problems in GAMS and the PATH solver," Journ. Econ. Dynam. Contr., vol. 24, no. 2, pp. $165-188,2000$

[25] S. Martín, Y. Smeers, and J. A. Aguado, "A stochastic two settlement equilibrium model for electricity markets with wind generation," IEEE Trans. Power Syst., vol. 30, no. 1, pp. 233-245, 2015.

[26] Ameren Electric Company, "Day ahead pricing used for billing real time price service," 2018. Available: https://www.ameren.com/account/ retail-energy

[27] N. Growe-Kuska, H. Heitsch, and W. Romisch, "Scenario reduction an scenario tree construction for power management problems," in Proc. Power Tech, Bologna, Italy, 2003. 


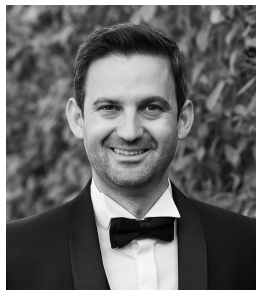

Dimitrios Thomas (S'08) received his diploma in Electrical and Computer Engineering from the Democritus University of Thrace, Greece and his $\mathrm{Ph} . \mathrm{D}$. degree in Electrical Engineering from the University of Mons, Mons, Belgium, in 2019. His research interests include applications of mathematical programming in energy and power systems, storage and flexibility services, local energy markets, optimization and game theory.

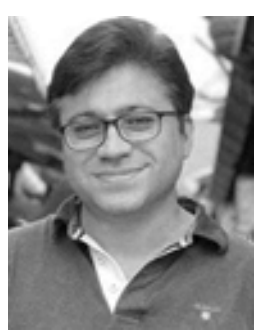

Jalal Kazempour (SM'18) received the Ph.D. degree in Electrical Engineering from the University of Castilla-La Mancha, Ciudad Real, Spain, in 2013. He is currently an Associate Professor with the Department of Electrical Engineering, Technical University of Denmark, Kgs. Lyngby, Denmark. His research interests include power and energy systems, electricity markets, optimization and game theory.

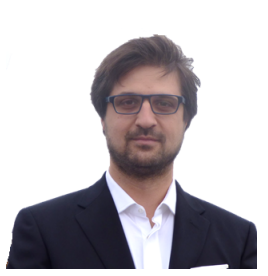

Athanasios Papakonstantinou received a BSc in Mathematics from the Aristotle University of Thessaloniki, Greece at 2005 and an MSc in Mathematics with Modern Applications for The University of York, UK at 2006. He got a $\mathrm{PhD}$ in Computer Science at 2010 from the School of Electronics and Computer Science in the University of Southampton in the UK. Since then he has been employed as a post-doctoral researcher in Copenhagen Business School (CBS) and the Technical University of Denmark (DTU). Today he is a tenure tracked researcher in the Dept. of Management Engineering in DTU. His research lies in the intersection of Economics, Optimization with applications in energy related topics.

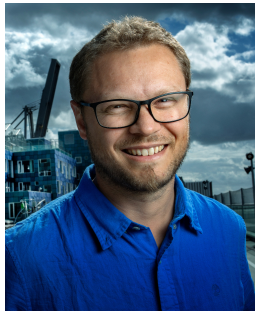

Pierre Pinson (SM'13, F'20) received the M.Sc. degree in applied mathematics from the National Institute for Applied Sciences, Toulouse, France, and the Ph.D. degree in energetics from Ecole des Mines de Paris, Paris, France. He is a Professor with the Centre for Electric Power and Energy, Department of Electrical Engineering, Technical University of Denmark, Lyngby, Denmark, also heading a group focusing on energy analytics and markets. His research interests include forecasting, uncertainty estimation, optimization under uncertainty, decision sciences, and renewable energies. He is the Editor-in-Chief for the International Journal of Forecasting.

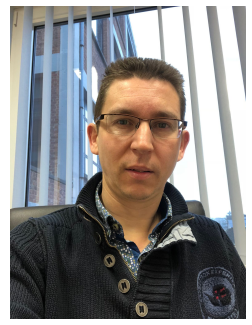

Olivier Deblecker (M'09) was born in 1971. He received the degree in civil electrical engineering and the $\mathrm{Ph} . \mathrm{D}$. degree in electrical engineering in 1995 and 2001, respectively. He is currently a Professor and Head of the Electrical Power Engineering Unit at the Faculty of Engineering, University of Mons (Belgium). His main fields of interest include power electronics and drives, as well as computational electromagnetics (particularly at intermediate frequencies). Recently, he has been conducting research in the field of micro-grids, energy management systems, renewables integration and optimization techniques. He has been a member of the European Power Electronics and Drives (EPE) Association since 2004 and IEEE since 2009.

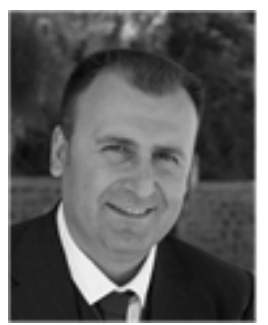

Christos S. Ioakimidis holds a B.Sc./Dipl.Ing. degree in mechanical engineering, Greece (1994), an M.S. degree in mechanical and aerospace engineering, USA (1996), a Ph.D. in mechanical and chemical engineering, U.K. (2001), and an MBA in financial engineering, Greece (2007). ERA-Chair Holder at Net Zero Energy Efficiency on City Districts, NZED Unit, (RIE/UMONS), Belgium and Programme Director of the MSc SMACCs. He is author/co-author of more than 207 articles in international journals and conferences under peerreview with a research and industrial experience (26-years) that includes 43 research projects (16.5ME) funded by regional, national and international governmental, public and private organizations. His research interests focus on complex transport/energy systems towards the Smart District/City concept including modelling, intelligent grids, systems integration (EVs, energy storage, renewable, mRES), energy efficiency, urban planning and design, sustainable mobility (e-mobility), innovation, entrepreneurship and green economy. $\mathrm{He}$ is an Associated Editor (AE) of IEEE Transactions on Intelligent Transportation Systems. 